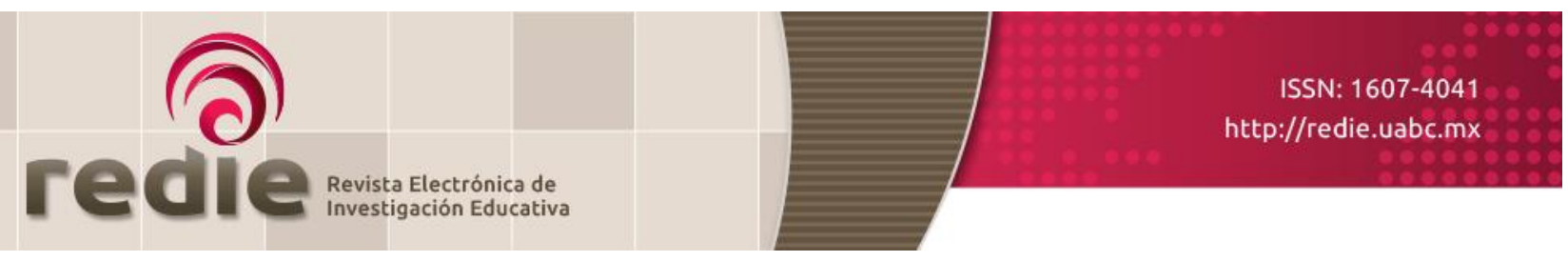

Vol. 19, Núm. 3, 2017

\title{
El uso de las tic en las prácticas académicas de los profesores de la Benemérita Universidad Autónoma de Puebla
}

\section{Use of ICT in Teachers' Academic Practices in the Benemérita Universidad Autónoma de Puebla}

Carolina Tapia Cortés (*) carolina tapia cortes@hotmail.com

Yadira Navarro Rangel (*) ynavarro44@gmail.com

Andrés Saúl de la Serna Tuya (*) asdelaserna@gmail.com

(*) Benemérita Universidad Autónoma de Puebla

(Recibido: 22 de febrero de 2016; Aceptado para su publicación: 17 de mayo de 2016)

Cómo citar: Tapia, C., Navarro, Y. y De la Serna, A. S. (2017). El uso de las tic en las prácticas académicas de los profesores de la Benemérita Universidad Autónoma de Puebla. Revista Electrónica de Investigación Educativa, 19(3), 115-125. https://doi.org/10.24320/redie.2017.19.3.1270

\section{Resumen}

Este artículo analiza cómo utilizan las Tecnologías de la Información y la Comunicación (TIC) en sus actividades académicas los profesores de tiempo completo, medio tiempo y por horas de la Benemérita Universidad Autónoma de Puebla (BUAP). En la investigación, de corte cuantitativo con un diseño correlacional, participaron 71 profesores, a quienes se les aplicó un cuestionario compuesto por 26 reactivos. Los resultados obtenidos indican que los profesores contratados por hora y en un rango de edad entre 25 a 38 años tienden a hacer un mayor uso de las TIc. En cuanto a las tecnologías más utilizadas por los profesores se encontraron: el correo electrónico, las presentaciones multimedia y las páginas web generales; mientras que las menos utilizadas son los foros, laboratorios virtuales y las videoconferencias.

Palabras clave: Tecnología de la Información, docencia, instituciones de educación superior

\begin{abstract}
This paper analyzes how full-time, part-time and hourly-contract teachers in the Benemérita Universidad Autónoma de Puebla (BUAP) use Information and Communication Technologies (ICTs) in their academic activities. In the quantitative and correlational research, a 26-item questionnaire was administered to 71 teachers. The results obtained show that hourly-contract teachers between 25 and 38 years of age tend to make greater use of IcTs. It was found that the most widely used technologies by teachers were e-mail, multimedia presentations and webpages in general, whereas forums, virtual laboratories and videoconferences were the least used.
\end{abstract}




\section{Introducción}

Nuestro tiempo está marcado por los acelerados cambios que conlleva el uso de las Tecnologías de la Información y la Comunicación (TIC), ante los cuales el contexto educativo no puede permanecer indiferente. La aparición de estas tecnologías ya son parte de nuestra vida diaria, en las actividades productivas y de tiempo libre (Karsenti, 2007). Adicionalmente, las TIC están permeando a todos los actores de la universidad, de manera particular el profesor -cuya práctica académica va a estar condicionada, sobre todo, por el conocimiento tecnológico que posee. Desde esta perspectiva, el uso de las TIC en las instituciones de educación superior (IES) ha pasado a ser un tema central en la agenda de las mismas. Lo anterior coincide con lo que el Conseil Supérieur de l'éducation (CSE, 2000) afirma:

La maîtrise du développement des technologies de l'information et de la communication en contexte éducatif est une exigence pour que chaque enseignant puisse maîtriser l'évolution de sa pratique et contribuer au devenir de la profession enseignante. ${ }^{1}$ (p. 74).

Bajo esta óptica, la utilización de las TIC en las prácticas académicas de los profesores no es un tema nuevo, una parte importante de la literatura nacional e internacional (Andion, 2011; Barrette, 2009; Coll, Mauri y Onrubia, 2008; Karsenti y Lira, 2011; Torres, Barona y García, 2010) analiza el impacto que han tenido las tecnologías en el contexto educativo; sin embargo, son minoría las investigaciones (Crovi, 2009) que exploran los factores que inciden en la utilización de las TIC en el contexto universitario.

La revisión de literatura en el ámbito de la introducción de las TIC en la universidad (Guzman, 2004; Lara, Zatarain y Cárdenas, 2013; Sunkel, Trucco y Espejo, 2014) señala también las bondades de la integración de las TIC en las prácticas académicas de los profesores con la finalidad de generar nuevos ambientes de aprendizaje mediados por tecnología.

Рara comprender la realidad en torno a la utilización de las TIC en las prácticas académicas de los profesores, se seleccionó una institución educativa desde la cual se pudiera analizar este proceso. En las últimas dos décadas la Benemérita Universidad Autónoma de Puebla (BUAP) ha iniciado transformaciones que la han posicionado como una de las mejores universidades del país (Execum, 2014). En este contexto, conscientes de la relevancia del tema y de los retos que enfrenta la universidad, se planteó una investigación con el objetivo de analizar cómo se utilizan las TIC más comunes, así como los factores que inciden en dichos usos por parte de los profesores de tiempo completo, medio tiempo y hora clase.

\subsection{La integración de las TIC en el ámbito universitario}

El uso de las TIC en el contexto universitario ha sido abordado en la literatura desde los modelos de integración de estas herramientas tecnológicas en la universidad. Una primera clasificación en torno al uso de las TIC en los procesos de integración dentro de las universidades la encontramos en Morais (2000) quien explica el uso a partir de la integración de las TIC en un modelo de cinco etapas: la pertinencia, el sentimiento de miedo, el uso personal, el nivel profesional y el nivel de enseñanza educativo en el que se enseña.

En la etapa pertinencia se establece que el profesor, previo a la integración de las TIC, debe estar convencido de la pertinencia pedagógica de las mismas. En la etapa sentimiento de miedo el profesor presenta variación en los usos, lo que en algunas ocasiones crea sensaciones de miedo, sin embargo éste puede eliminarse cuando el profesor está convencido de las oportunidades educativas de las herramientas. En la etapa uso personal se observa que las tecnologías son utilizadas para fines personales (comunicación con los familiares, elaboración de documentos e investigación sobre temas de interés personal, los cuales no tienen relación con las necesidades profesionales). En la etapa uso profesional el profesor integra el uso de las TIC para actividades propias de su actividad laboral (comunicación en colegas, planificación de las clases) y, por último, en la etapa nivel de enseñanza el profesor utiliza las

\footnotetext{
${ }^{1}$ Nota del editor. Dominar el desarrollo de Tecnologías de Información en un contexto educativo es un requisito que cada profesor puede controlar en la evolución de su práctica y contribuir al futuro de la profesión docente.
} 
tecnologías con el propósito de potenciar el proceso de enseñanza y aprendizaje.

De acuerdo con las cinco etapas presentadas, es importante señalar que el orden de las mismas es progresivo y común, incluso podría decirse que es conveniente que los profesores crucen gradual y sistemáticamente de cada una de las etapas.

Una clasificación más global de los usos de las tecnologías es presentada por Eduteka (2008), que establece seis niveles de integración: en los primeros tres niveles (1. preintegración, 2. instrucción dirigida y 3. integración básica) el profesor utiliza las tecnologías como medio de información y comunicación; en los siguientes dos niveles (4. medio y 5. avanzado) el profesor integra las TIC en su práctica docente a través del currículo y es hasta el nivel 6 (integración experta) cuando el profesor desarrolla y promueve ambientes de aprendizaje mediados por las TIC.

Por otro lado, de manera más reciente Surman y Reilly (2005) diferencian tres dimensiones que refieren la integración de las tecnologías a través del uso de estas herramientas:

- Dimensión acceso: el acceso está vinculado a la disponibilidad de hardware y la conexión a Internet.

- Dimensión adopción: las tecnologías son utilizadas como medios de información y la mayoría de los usuarios utilizan las TIC como consumidores.

- Dimensión apropiación: el uso de las TIC responde a un objetivo establecido por los usuarios y responde a necesidades específicas de una organización. Es en la apropiación cuando los usuarios llegan a ser productores y participantes de las tecnologías.

Otro enfoque más global e integrador de la incorporación de las TIC es presentado por Saga y Zmud (1994), quienes definen un modelo a partir de la aceptación de las tecnologías con base en las siguientes variables:

- Creencias sobre efectividad: vincula las creencias sobre la utilidad de estas herramientas y las intenciones de uso, es decir, los individuos pueden intentar usar la tecnología porque perciben que resulta útil y socialmente importante, aun cuando no cuenten con una actitud favorable hacia ella.

- Conocimiento: la utilidad de las tecnologías obedecerá al conocimiento que el individuo tiene de las mismas, por lo tanto se tiene que a mayor conocimiento hay una mayor integración de las TIC, y viceversa.

- Intervención administrativa: son las acciones o estrategias (disponibilidad de infraestructura tecnológica, capacitación e incentivos) que realiza la administración con la finalidad de motivar el uso de la tecnología.

- Participación del usuario: son las actividades desempeñadas por los usuarios con relación al uso de las tecnologías.

De manera complementaria, estos autores añaden que se dan dos fases en la implementación de la tecnología:

1. Rutinización, en esta fase el uso de una tecnología forma parte de las actividades diarias de los usuarios, es decir se institucionalizan estas herramientas.

2. Infusión, esta sucede cuando el uso de una tecnología atraviesa por un proceso preliminar de forma reflexiva y global. 
Un análisis de los modelos de integración emitidos por los autores estudiados los clasifica en tres niveles:

1. Nivel básico: el uso de las tecnologías está vinculado al acceso y disponibilidad de estas herramientas y el profesor sólo las utiliza como consumidor y medio de información y comunicación.

2. Nivel intermedio: en este nivel se observa una intervención de la institución con la finalidad de promover la utilización de las TIC, se espera que el profesor tenga conocimiento de las tecnologías y las integre de forma progresiva en sus actividades académicas.

3. Nivel avanzado: éste sucede cuando el profesor integra las tecnologías en su práctica docente como medio para optimizar el proceso de enseñanza-aprendizaje a través del currículo, es decir, genera ambientes de aprendizaje mediados por tecnología, se vuelve productor y participante de la tecnología.

Los modelos de integración de la tecnología son un factor a tomar en cuenta en la comprensión del uso de las TIC, ya que determinan un proceso de incorporación adecuado y efectivo, por lo que su investigación y seguimiento es urgente. En este apartado se evidencia que las investigaciones en torno a la integración de las TIC aportan elementos de reflexión, al focalizarse en las dimensiones asociadas al uso, las investigaciones reflejan los diferentes niveles de integración de estas herramientas, así como las condiciones necesarias para su utilización.

\subsection{La orientación del uso de las TIC: ¿información o comunicación?}

Superar el obstáculo del acceso y disponibilidad de las TIC atiende las dos primeras condiciones para una integración e impacto efectivo de las TIC en la educación; sin embargo, es necesario avanzar hacia la siguiente condición e indagar cómo y cuáles son los modos de uso de estas tecnologías por parte de los profesores.

Bajo esta concepción, el profesor es el principal agente educativo para promover el aprovechamiento de estas herramientas, por lo que es necesario pasar de la aceptación de las mismas a una utilización vinculada al ámbito educativo. A este respecto, Torres y Lara (2013) señalan que el uso de las TIC por parte de los profesores ha quedado en el plano de la información, considerando únicamente el aspecto unidireccional, es decir, que va del profesor al alumno, dejando de lado el plano de comunicación, el cual implica el aspecto bidireccional.

En un sentido similar Cabero (2005) señala que los principales usos de las TIC por parte de profesores están orientados a motivar a los alumnos al acceso y presentación de información, dejando en menos porcentaje los usos que refieren innovación, y añade que las tecnologías no se pueden limitar a la transmisión de información, es necesaria la información a todos los niveles, iniciar la comunicación entre personas que trabajan en el centro educativo, seguir con la gestión y administración del centro, hasta llegar a las actividades de investigación y formación.

En este contexto, el tema uso de las TIC por parte de profesores exige finalmente profundizar en los modos de utilización de las tecnologías que van orientados al uso estratégico, innovador y con fines específicos, lo anterior permitirá cambios verdaderos en los procesos de enseñanza-aprendizaje.

\section{Metodología}

Se recurrió a un proceso de investigación cuantitativa a partir del diseño y aplicación de un cuestionario. El instrumento consistió en cinco apartados en relación con: a) aspectos sociodemográficos; b) el contexto laboral; c) la percepción de las TIC, d) el uso de las TIC y e) disponibilidad de las TIC. En esta investigación únicamente se analizan los reactivos relativos al uso de las TIC. Cabe señalar que en la muestra sólo se incluye a profesores de la modalidad presencial. El criterio de muestreo utilizado fue no probabilístico y éste, a su vez, tuvo como base el intencionado. Se aplicó el cuestionario a 20 profesores 
de Ingeniería, 16 de Humanidades, 14 del área de la Salud y 21 de Administración. En el estudio participaron 39 hombres (54.9\%) y 32 mujeres (45.1\%), cuyo rango de edad fluctuó entre 26 y 68 años, teniendo una media de 45 años. El procesamiento de los datos se realizó a través del análisis factorial exploratorio por medio del paquete computacional SPSS. El análisis permitió en un primer momento realizar la descripción de datos sociodemográficos, contexto laboral y uso de las TIC; posteriormente se procedió al análisis factorial, que permitió la agrupación de las respuestas de los informantes en factores, finalmente se realizaron pruebas ANOVA para contrastar estos últimos con las variables directas (sexo, edad, antigüedad laboral, estado civil, condición laboral, unidad de adscripción y máximo grado de estudios).

\section{Resultados}

Los resultados se presentan de forma secuencial en dos momentos: a) Primero. Análisis descriptivo de las variables adicionales (aspectos sociodemográficos, y frecuencia en el uso de las TIC), en la segunda parte se presentan los descriptivos de los modos de uso de las TIC; y b) Segundo. Análisis Factorial Exploratorio mediante la técnica de componentes principales con rotación VARIMAX.

\subsection{Primer momento. Análisis descriptivo}

La media de edad es de 45 años y el nivel mínimo de estudio de los profesores es la Maestría. La media de antigüedad se presenta en 15 años. En la modalidad para impartir clases se concentraron en la presencial. El tiempo dedicado semanalmente a la realización de actividades con el uso de las TIC se concentró en el rango de 11 a 20 horas y, en general, son los hombres los que más las utilizan (Torres, Tapia y Barona, 2013).

Los resultados presentados en la figura 1 reflejan que los profesores tienen un uso limitado de las TIC, ya que sólo el $52 \%$ refirió hacer uso de ellas de forma constante o siempre. Del mismo modo, se encontró que es el correo electrónico la herramienta más utilizada, el 89\% de los profesores señaló que la utiliza constantemente o siempre, seguido de presentaciones multimedia (78\%), páginas web generales (70\%), portal BUAP (70\%) y servicios de comunicación (59\%); mientras que las menos utilizadas son las redes sociales (46\%), suscripción a listas de correos, plataformas (39\%), weblogs (38\%), foros (34\%), laboratorios virtuales (34\%) y videoconferencias (29\%). Estos resultados evidencian que los profesores tienden a utilizar las tecnologías enfocadas a la transmisión de información y que se sitúa en la actualidad por algunos autores (Torres y Lara, 2013; Cabero, 2005) como la de mayor porcentaje de uso por parte de los profesores. Asimismo, las tecnologías más utilizadas por los profesores se enmarcan en la perspectiva instrumental (Joüet, 1993; Lara, Zatarin y Cárdenas, 2013). Finalmente, las tecnologías que se sitúan en ambientes virtuales de aprendizaje permanecen como las menos utilizadas por los profesores.

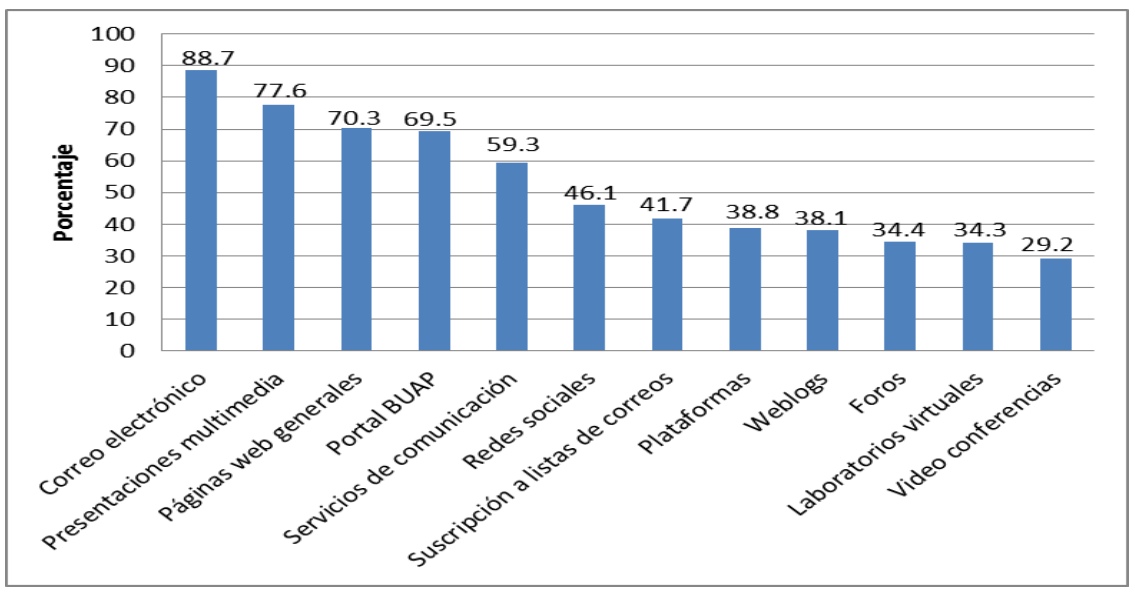

Figura 1. Modos de uso de las TIC 


\subsection{Segundo momento. Análisis factorial}

En este apartado se presenta la conformación de cada uno de los factores encontrados, así como sus ítems y el coeficiente alfa (ver tabla I). Los dos factores identificados con sus respectivos ítems y el coeficiente alfa con base en el procesamiento antes mencionado fueron:

- Primer factor. Uso de las tIC en entornos virtuales, este factor refiere el uso de foros, videoconferencias, laboratorios virtuales, plataformas, servicios de redes sociales y weblogs.

- Segundo factor: uso de las tIC para la comunicación-información, comprende el uso del correo electrónico, la suscripción a listas de correo y las páginas web generales.

Tabla I. Componentes de los factores de usos de las tic en las prácticas académicas

\begin{tabular}{c|c|c}
\hline Factor & Reactivos & $\begin{array}{c}\text { Coeficiente } \\
\text { Alfa de Cronbach }\end{array}$ \\
\hline $\begin{array}{c}\text { Uso de tIc en } \\
\text { entornos } \\
\text { virtuales }\end{array}$ & $\begin{array}{c}\text { Foros } \\
\text { Weblogs } \\
\text { Laboratorios virtuales } \\
\text { Videoconferencias } \\
\text { Servicios de redes sociales } \\
\text { Portal de la buap }\end{array}$ & 0.871 \\
\hline $\begin{array}{c}\text { Uso de tic } \\
\text { para la } \\
\text { comunicación- } \\
\text { información }\end{array}$ & $\begin{array}{c}\text { Correo electrónico } \\
\text { Puscripinas web generales a listas de correo }\end{array}$ & 0.590 \\
\hline
\end{tabular}

Es conveniente aclarar que en el modelo teórico, los recursos correo electrónico, páginas web generales, suscripción a listas de correos y portal de la BUAP corresponden al uso de TIC para la comunicación e información, sin embargo la utilización del portal de la BUAP está marcando la diferencia, como lo indica el análisis factorial.

Se esperaría que en el nivel uso de las TIC para la comunicación e información los profesores hagan uso unidireccional o de meros consumidores de la información, mientras que en el nivel uso de TIC en ambientes virtuales ya son "prosumidores", es decir, interactúan consumiendo y produciendo información.

\subsection{Pruebas de contraste}

Al aplicar la prueba de análisis de varianza (ANOVA) entre los factores y algunas variables directas se obtuvieron los siguientes resultados: el contraste de la variable tipo de contrato con el factor uso comunicación-información resultó significativa (.079) por lo que podemos decir que el tipo de contrato marca la diferencia en el uso que hacen los profesores de estas herramientas. De la misma forma, el contraste del factor anterior con la variable rango de edad resultó significativo (.028), de lo que interpretamos que la edad de los profesores influye en el uso que se hace de las Tic que refieren el uso del correo electrónico, suscripción a listas de correo y páginas web generales. A su vez, se muestra que el contraste del factor uso docencia con la variable tipo de contrato es significativo, por tanto, es posible afirmar que el tipo de contrato influye en el uso de las TIC en la docencia. 
Las figuras 2 y 3 presentan los contrastes realizados entre los factores encontrados y las variables (tipo de contrato y edad).

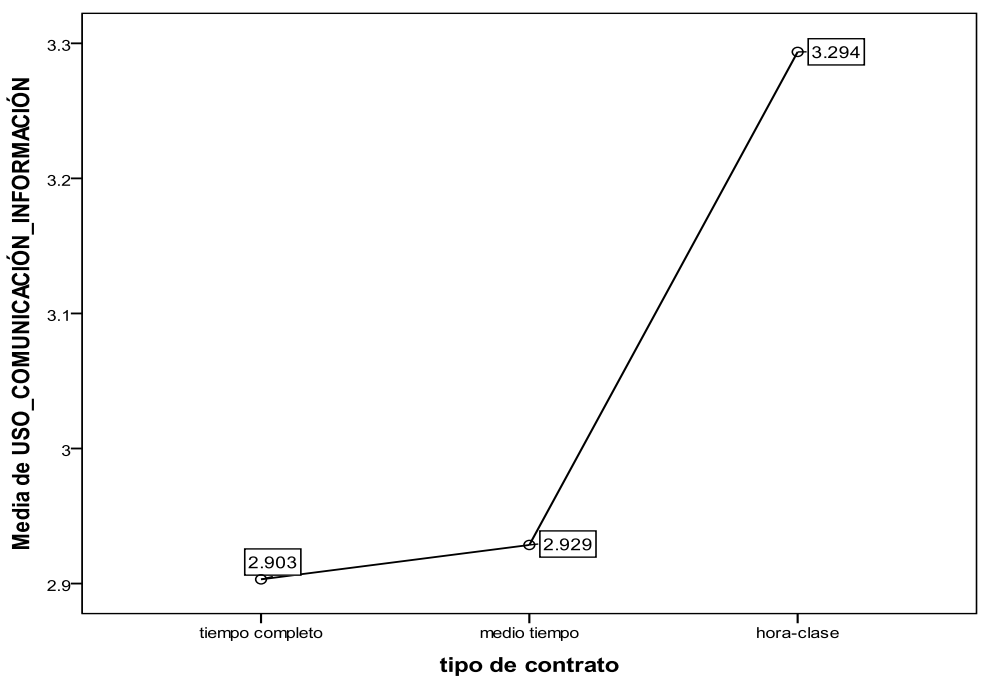

Figura 2. Prueba ANova del factor uso comunicación-información con la variable tipo de contrato Escala: 1 nunca, 2 a veces, 3 constantemente, 4 siempre.

En la figura 2 se aprecia la implicación del factor uso comunicación-información con la variable tipo de contrato, los datos iniciales plantean aspectos interesantes. Un primer aspecto que resalta es que los profesores contratados por horas hacen un mayor uso de las TIC asociadas al correo electrónico, suscripción a listas de correo y páginas web generales. Un segundo aspecto es que los profesores de tiempo completo y medio tiempo hacen un menor uso de estas tecnologías y se observa que no hay diferencias significativas entre ambos grupos, lo cual apunta hacia un comportamiento similar de utilización de dichas tecnologías, esto probablemente se debe a que ambos grupos cuentan con condiciones laborales similares, es decir: estabilidad laboral, menos rotación y dedicación exclusiva.

Al revisar la edad promedio de los profesores (tabla II) destaca que los profesores contratados por horas son más jóvenes en comparación con los profesores de medio tiempo y tiempo completo.

Tabla II. Edad promedio de los profesores

\begin{tabular}{cc}
\hline Tipo de contratación & Edad-promedio \\
\hline Tiempo completo & 50 años \\
Medio tiempo & 41 años \\
Hora-clase & 37 años \\
\hline
\end{tabular}

Los resultados obtenidos parecen validar, al menos parcialmente que los profesores contratados por horas y jóvenes, suelen hacer un mayor uso de las TIC asociadas a la información y comunicación (correo electrónico, suscripción a listas de correo y páginas web generales). Además, al considerar que dichas tecnologías son las más fáciles respecto al acceso, disponibilidad y uso, es de llamar la atención que los resultados revelan un uso reducido de estas tecnologías por parte de profesores de medio tiempo y tiempo completo.

Cabe preguntarse, entonces, ¿cómo incide el tipo de contratación de un profesor en el uso de las TIC?, actualmente la definitividad se logra después de 5 años de servicio ininterrumpido y para poder concursar por un ascenso o promoción laboral se requiere de la misma. Las nuevas contrataciones se hacen en función de las necesidades de cubrir grupos y son por horas y período determinado. 
Los contratos por plazas altas (tiempo completo, categoría titular, etc.) exigen doctorado y han sido orientados a la investigación, es decir, exige un alto perfil.

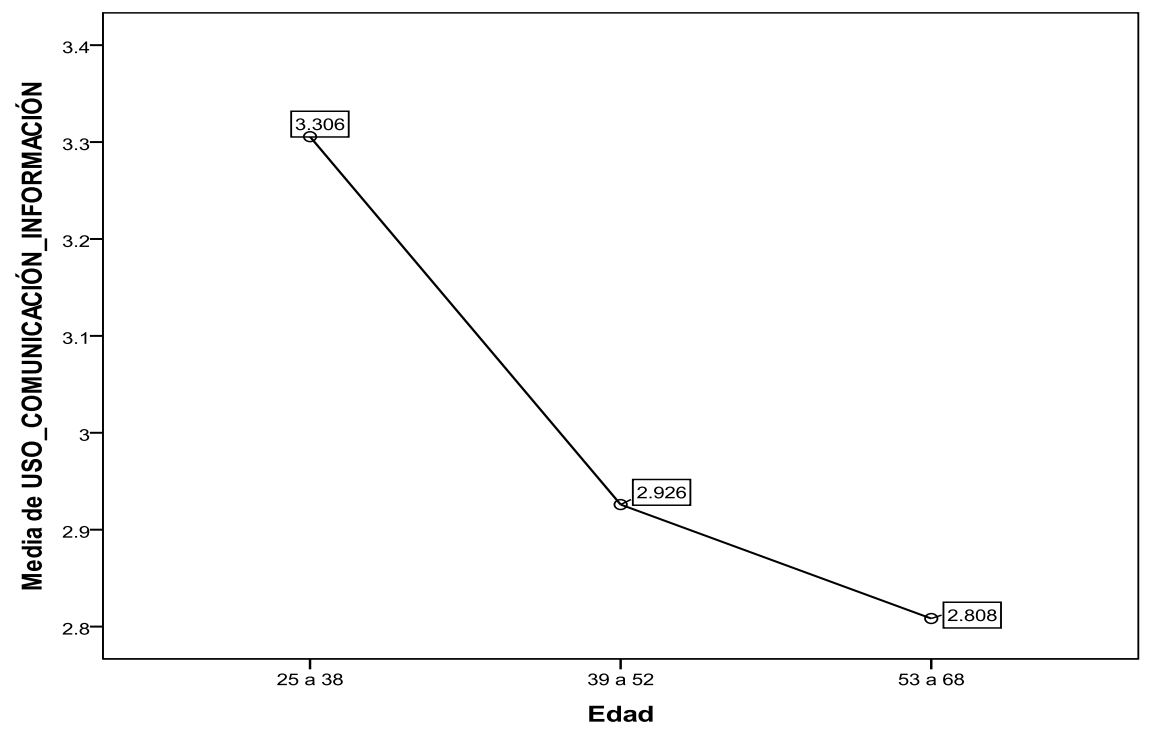

Figura 3. Prueba ANova del factor uso comunicación-información con la variable rango de edad Escala: 1 nunca, 2 a veces, 3 constantemente, 4 siempre.

En la figura 3 se aprecia que el factor uso comunicación-información está inversamente relacionado con la edad de los profesores y de acuerdo con las medias los profesores que se encuentran en un rango de edad de 25 a 38 años (3.30) son los que más utilizan las TIC relacionadas con la comunicación-información, seguido de los profesores ubicados entre los 39 a 52 años (2.92) y con menos frecuencia de uso se encuentran los profesores de 53 a 68 años de edad (2.80). Lo anterior implica que a menor edad de los profesores se observa un mayor uso de las TIC vinculadas a la comunicación e información. Los resultados de este contraste nos permiten confirmar hallazgos que otras investigaciones postulan (Zubieta, Bautista y Quijano, 2012), es decir, que los profesores de mayor edad tienden a hacer un menor uso de la tecnología.

\section{Conclusiones}

La integración de las TIC en las universidades es una de las principales líneas de cambio por las ventajas que diferentes organismos (Banco Mundial, 2003; UNESCO, 2009) han señalado. En el caso de la BUAP se han tenido avances significativos en materia de infraestructura tecnológica e intervención administrativa, esta última señalada por Saga y Zmud (1994) como una de las dos principales variables para el éxito en la implementación de tecnologías.

Del mismo modo la BUAP, a través del discurso expresado en los documentos estratégicos (Modelo Universitario Minerva, informe de labores y programas institucionales de desarrollo educativo correspondientes a la anterior y a la actual administración), ha marcado la pauta para seguir en busca de una integración y uso efectivo de las TIC, reflejando el esfuerzo realizado para lograr la integración de estas herramientas en el ámbito educativo, aunque al parece los efectos no han sido notables, pues los resultados indican que el uso de las TIC se centra en el correo electrónico, presentaciones multimedia, páginas web generales y portal BUAP, es decir, un uso instrumental o técnico y orientado a la información, dejando de lado la dimensión comunicación (Cabero, 2005; Torres y Lara, 2013). Estos datos confirman hallazgos de otros estudios que postulan lo mismo en torno al tema (Zubieta, Bautista y Quijano, 2012; Bernet y Karsenti, 2013). 
Con base en el análisis de los procesos de integración de las TIC en el ámbito universitario (Eduteka, 2008; Surman y Reilly, 2005) es posible señalar que los profesores se encuentran en la dimensión adopción o integración básica, es decir, los profesores utilizan las tecnologías como consumidores.

Otro aspecto a considerar en los resultados, es lo señalado por Esparza (2016), quien menciona que la edad promedio de la planta docente de esta casa de estudios es de 56 años, lo que apunta hacia dos panoramas posibles:

a) El primero sería Laissez fair-Laissez passer (dejar hacer-dejar pasar), es decir, que los docentes de mayor edad se jubilen y que esos puestos sean ocupados por jóvenes altamente calificados que en la mayoría de los casos ya fueron formados y educados en el uso de las TIC. Ello apuntaría a una posición extremadamente pasiva de la universidad para la formación de sus cuadros o planta docente.

b) El segundo sería una posición proactiva, es decir intervencionista, y que refiere acciones y estrategias proactivas, lo que en palabras de Saga y Zmund (1994) se trata de la rutinización de actividades mediante la utilización de las TIC. Ejemplo de ello podría ser el uso del portal BUAP para calificar a los estudiantes, procesos de evaluación y otras acciones de gestión académica y administrativa.

Si bien ya se da una rutinización de actividades a un nivel muy básico en la BUAP, cabe señalar que estas acciones no tienen un efecto directo en la docencia o lo que sucede en el aula.

Sobre los elementos que inciden en el uso de las TIC, cabe señalar nuestra coincidencia con el posicionamiento de Zubieta, Bautista y Quijano (2012), respecto a que los profesores de mayor edad tienden a hacer un menor uso de la tecnología y viceversa. A menor edad mayor uso, en este caso los profesores en un rango de edad de 25 a 38 años. En este sentido, se evidencia que los profesores que hacen un menor uso de las tecnologías asociadas a la información y comunicación son los que se ubican en un rango de edad entre 53 a 68 años. Con este resultado podemos deducir la presencia de una idea subyacente en los profesores de mayor edad y que considera, al menos aparentemente, que no se han creado necesidades de uso de dichas tecnologías en las prácticas académicas, principalmente en la docencia.

Los posibles motivos que los profesores mencionaron para no hacer uso de las TIC se relacionan esencialmente con lo que señalan Mata y Acevedo (2010), respecto a las dificultades que encuentran frente al uso de las TIc: falta de tiempo para la formación en estas herramientas, una mala estructuración organizativa de las instituciones educativas, una cierta actitud de reserva hacia el cambio, la percepción de que los estudiantes conocen y utilizan más las TIC que ellos, lo que rompe con la concepción tradicional de que el maestro es el centro del conocimiento.

Otro factor presente en la incidencia del uso de las TIC se asocia al tipo de contratación, los profesores que tienen contrato hora clase son los que están haciendo un mayor uso de las tecnologías, con este resultado podemos deducir que los esfuerzos y estrategias que la institución ha llevado a cabo para la incorporación de las tecnologías se reflejan aparentemente en los profesores contratados por hora, prueba de lo anterior es que son este tipo de profesores los que asisten a los cursos de capacitación y actualización. Lo anterior pone en evidencia la existencia de otros factores relacionados al contexto educativo (en este caso el tipo de contratación de un profesor) que inciden en la utilización de las Tic.

Los resultados obtenidos hacen suponer que son dos variables las que están incidiendo en el uso de las TIC: la edad y el tipo de contrato, sin embargo, al revisar detenidamente la última variable, es posible deducir que el Programa Institucional de Evaluación Académica (PIEVA) procedimiento mediante el cual se valora, desde la perspectiva del estudiante, el desempeño de la práctica docente y actividad tutorial por el que atraviesan los profesores en la universidad cada fin de cuatrimestre y que califica entre otros aspectos la utilización de tecnologías, hace suponer que los profesores contratados por horas buscan obtener mejores calificaciones a través de este rubro, con la finalidad de renovar su contratación. Cabe entonces preguntarse si la utilización de las tecnologías está ligada a otros factores del contexto 
universitario (procesos de evaluación, nivel de carga docente, condiciones laborales, etc.).

Finalmente, lo que este estudio arroja es que existen variables contextuales que deben ser analizadas con el fin de plantear programas de intervención que no se limiten a la capacitación docente, sino que generen ambientes propicios que induzcan al docente a usar las TIC para su formación y actualización, sus actividades de investigación y difusión, es decir, que los procesos académicos se hagan más eficientes mediante el uso de las TIC. Un primer paso puede ser el uso generalizado de la plataforma institucional para alojar los cursos.

\section{Referencias}

Andión, M. (2011). La apropiación social de las TIC en la educación superior. Revista Reencuentro, 62, 5-9. Recuperado de http://bidi.xoc.uam.mx/tabla contenido fasciculo.php

Banco Mundial. (2003). ICT and MDGs: A World Bank Perspective. Washington: Autor.

Barrette, C. (2009). Métarecherche sur les effets de l'intégration des TIC en pédagogie collégiale [Metabuscador sobre los efectos de la integración de las TIC en la enseñanza universitaria]. Revue Internationale Des Technologies En Pédagogie Universitaire, 6(2-3), 18.

http://doi.org/10.7202/1000008ar

Bernet, E. y Karsenti, T. (2013). Modes d'intégration et usages des TIC au troisième cycle du primaire : une étude multicas [Métodos de integración y uso de las TIC en el tercer ciclo de primaria]. Éducation Et Francophonie, 41(1), 45. Recuperado de https://www.erudit.org/revue/ef/2013/v41/n1/1015059ar.pdf

Cabero, J. (2005). Las TIC y las universidades: retos, posibilidades y preocupaciones. Revista de la Educación Superior, 34(135), 77-100.

Coll, C., Mauri, T. y Ontubia, J. (2008). Análisis de los usos reales de las TIC en contextos educativos formales: una aproximación sociocultural. Revista Electrónica de Investigación Educativa, 10(1). Recuperado de http://redie.uabc.mx/redie/article/view/177

Conseil Supérieur de l'Éducation. (2000). Éducation et nouvelles technologies. Pour une intégration réussie dans l'enseignement et l'apprentissage. Rapport annuel 1999-2000 sur l'état et les besoins de l'éducation [La educación y las nuevas tecnologías. Para una integración exitosa en la enseñanza y el aprendizaje. Informe anual 1999-2000 sobre el estado y las necesidades de la educación]. Recuperado de https://www.cse.gouv.qc.ca/fichiers/documents/publications/RapportsAnnuel/rapann00.pdf

Crovi, D. (2009). Acceso, uso y apropiación de las TIC en comunidades académicas. Diagnóstico en la UNAM. México: Plaza y Valdés.

Eduteka. (2008). Un modelo para integrar las TIC en el currículo. Recuperado de http://www.eduteka.org/tema mes.php3

Esparza, A. (2016, enero 26). Solicita BUAP presupuesto para 200 plazas de profesores. Recuperado de http://www.oem.com.mx/elsoldepuebla/notas/n3658320.htm

Execum. (2014). Estudio comparativo de las universidades mexicanas. Explorador de datos. Recuperado de http://www.execum.unam.mx

Guzmán, M. A. (2004). Estudio sobre los usos didácticos, procesos formativos y actitudes de los docentes universitarios en relación a internet. Revista Iberoamericana de Educación. Recuperado de http://www.rieoei.org/deloslectores/633Guzman.pdf

Joüet, J. (1993). Pratiques de la médiation [Prácticas de la mediación]. Réseaux, 60(6). 
Karsenti, T. y Lira, M. L. (2011). La importancia de la motivación y las habilidades computacionales de los futuros profesores en el uso de las TIC. Revista Iberomericana de Educación Superior, 2(3), 116-129.

Karsenti, T. (2007). Les TIC bouleversent l'enseignement. CHAIRE de recherche du Canada sur les technologies de l'information et de la communication (TIC) en éducation [Las TIC interrumpen la enseñanza. Grupo de Investigación de Canadá de las TIC en la educación]. Recuperado de http://karsenti.scedu.umontreal.calchaire.php

Lara, J., Zatarain, C. y Cárdenas, A. (2013). Proceso de apropiación de las tic por parte de los docentes. En S. A. T. Velandia y J. de J. Lara (Coords.), Usos y apropiación de las TIC: experiencias en el proceso educativo (pp. 17-52). Recuperado de http://sistemanodalsinaloa.gob.mx/archivoscomprobatorios/ 13 libro/63.pdf

Mata, A. I. y Acevedo, A. C. (2010). La actitud de los profesores hacia el uso de las TIC. Revista Investigación y Posgrado, 25(2-3), 143-180.

Morais, M. A (2000). Les cinq niveaux d'appropriation des TIC dans les pratiques pédagogiques chez les enseignantes et enseignants [Los cinco niveles de apropiación de las TIC en las prácticas de enseñanza entre los maestros]. Documento no publicado. Nouveau Brunswick, Université de Montreal.

Saga, V. L. y Zmud, R. W. (1994). The nature and determinants of IT-acceptance, routinization, and infusion, en L. Levine (Ed.), Diffusion, transfer and implementation of information technology (pp. 67-86). Holanda: Elsevier.

Sunkel, G., Trucco, D. y Espejo, A. (2014). La integración de las tecnologías digitales en las escuelas de América Latina y el Caribe Una mirada multidimensional. Chile: Comisión Económica para América Latina y el Caribe.

Surman, M. y Reilly, K. (2005). Apropiarse de Internet para el cambio social. Hacia un uso estratégico de las nuevas tecnologías por las organizaciones transnacionales de la sociedad civil. Informe de investigación.

Torres, S., Tapia, C. y Barona, C. (2013). Infraestructura tecnológica y apropiación de las TIC en la Benemérita Universidad Autónoma de Puebla. Estudio de caso, en Usos y apropiación de las TIC: experiencias en el proceso educativo. México: Universidad Autónoma de Sinaloa: Juan Pablos Editor.

Torres, S., Barona, C. y García, O. (2010). Infraestructura tecnológica y apropiación de las TIc en la universidad autónoma del estado de morelos: Estudio de caso. Perfiles Educativos, 32(127), 105-127. Recuperado de http://www.iisue.unam.mx/perfiles2/article/view/18881

Torres, S. A. y Lara, J. de J. (2013). Usos y apropiación de las TIC: experiencias en el proceso educativo. México: Universidad Autónoma de Sinaloa-Juan Pablos.

UNESCO. (2009). Declaración mundial sobre la educación superior. Recuperado de http://www.unesco.org/education/educprog/wche/declaration spa.htm

Zubieta, J., Bautista, T. y Quijano, A. (2012). Aceptación de las tIC en la docencia. México: Universidad Autónoma de México-Porrúa. 